\title{
Multiwavelength Observations of the February 1996 High-Energy Flare in the Blazar 3C 279
}

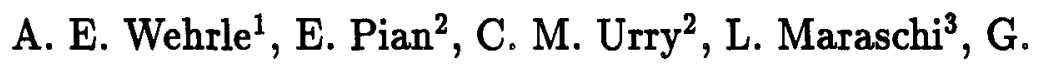
Ghisellini $^{4}$, R. C. Hartman ${ }^{5}$, G. M. Madejski ${ }^{5}$, F. Makino ${ }^{6}$, A. P. Marscher ${ }^{7}$, I. M. McHardy ${ }^{8}$, J. R. Webb ${ }^{9}$, G. S. Aldering ${ }^{10}$, M. F. Aller ${ }^{11}$, H. D. Aller ${ }^{11}$, D. E. Backman ${ }^{12}$, T. J. Balonek ${ }^{13}$,

P. Boltwood ${ }^{14}$, J. Bonnell ${ }^{5}$, J. Caplinger ${ }^{15}$, A. Celotti ${ }^{16}$, W. Collmar ${ }^{17}$, J. Dalton ${ }^{12}$, A. Drucker ${ }^{12}$, R. Falomo ${ }^{18}$, C. E. Fichtel $^{5}$, W. Freudling ${ }^{17}$, W. K. Gear ${ }^{19}$, N. Gonzalez-Perez ${ }^{20}$, P. Hall ${ }^{21}$, H. Inoue ${ }^{6}$, W. N. Johnson ${ }^{22}$, M. R. Kidger ${ }^{20}$, R. I. Kollgaard $^{23}$, Y. Kondo ${ }^{5}$, J. Kurfess ${ }^{22}$, A. J. Lawson ${ }^{8}$, B. $\mathrm{McCollum}^{5}$, K. McNaron-Brown ${ }^{22}$, D. Nair ${ }^{9}$, S. Penton ${ }^{24}$, J. E. Pesce $^{2}$, M. Pohl ${ }^{17}$, C. M. Raiteri ${ }^{25}$, M. Renda ${ }^{12}$, E. I. Robson $^{19,26}$, R. M. Sambruna ${ }^{5}$, A. F. Schirmer ${ }^{13}$, C. Shrader ${ }^{5}$, M. Sikora ${ }^{35}$, A. Sillanpää ${ }^{27}$, P. S. Smith ${ }^{28}$, J. A. Stevens ${ }^{12}$, J. Stocke $^{24}$, L. O. Takalo ${ }^{27}$, H. Teräsranta ${ }^{29}$, D. J. Thompson ${ }^{5}$, R. Thompson ${ }^{15}$, M. Tornikoski ${ }^{29}$, G. Tosti ${ }^{30}$, P. Turcotte ${ }^{12}$, A. Treves $^{31}$, S. C. Unwin ${ }^{32}$, E. Valtaoja ${ }^{29}$, M. Villata ${ }^{25}$, S. J. $\mathrm{Wagner}^{33}$, W. Xu $\mathbf{X}^{1}$, A. C. Zook ${ }^{34}$

${ }^{1}$ Infrared Processing Analysis Center, MC 100-22, California Institute of Technology, Pasadena, CA 91125

${ }^{2}$ Space Telescope Science Institute, 3700 San Martin Drive, Baltimore, MD 21218

${ }^{3}$ Osservatorio Astronomico di Brera, Via Brera 28, I-20121 Milan, Italy

4 Osservatorio Astronomico di Brera, Via Bianchi 46, I-22055 Merate (Lecco), Italy

${ }^{5}$ NASA/Goddard Space Flight Center, Greenbelt, MD 20771

${ }^{6}$ ISAS, 3-1-1, Yoshinodai, Sagamihara, Kanagawa 229, Japan

${ }^{7}$ Department of Astronomy, Boston University, 725 Commonwealth Avenue, Boston, MA 02215

${ }^{8}$ Department of Physics, University of Southampton, Southampton SO9 5NH, United Kingdom

${ }^{9}$ Department of Physics, Florida International University, University Park, Miami, FL 
${ }^{10}$ University of Minnesota, Department of Astronomy, 116 Church St., SE Minneapolis, $M N 55455$

${ }^{11}$ University of Michigan, Physics and Astronomy, 817 Dennison Building, Ann Arbor, $M I \$ 8109$

${ }^{12}$ Franklin \& Marshall College, Physics \& Astronomy Department, P.O. Box 3003, Lancaster, PA 17604-3003

${ }^{13}$ Colgate University, Department of Physics 8 Astronomy, 19 Oak Dr., Hamilton, NY 19346-1998

${ }^{14} 1655$ Main St., Stittsville, Ontario, Canada K2S 1N6

${ }^{15}$ IUE Data Analysis Center, NASA/GSFC, Greenbelt, MD 20771

${ }^{16}$ ESTEC, Astroph. Div. Space Sci. Dept., Postbus 299, 2200 Noordwijk, The Netherlands

${ }^{17}$ Max Planck-Institut für Extraterrestrische Physik, Giessenbachstrasse, D-85740

Garching bei München, Germany

${ }^{18}$ Osservatorio Astronomico di Padova, Via Osservatorio 5, I-35122 Padova, Italy

${ }^{19}$ Centre for Astrophysics, University of Central Lancashire, Preston, PR1 2HE, United Kingdom

${ }^{20}$ Instituto de Astrofísica de Canarias, E-38200, La Laguna, Tenerife, Spain

${ }^{21}$ Steward Observatory, University of Arizona, Tucson AZ 85721

${ }^{22}$ Naval Research Lab., 4555 Overlook Av., SW, Washington, DC 20375-5952

${ }^{23}$ Department of Astronomy and Astrophysics, The Pennsylvania State University, University Park, PA 16802

${ }^{24}$ University of Colorado, JILA, Campus Box 440, Boulder, CO 80309-0440

${ }^{25}$ Osservatorio Astronomico di Torino, Strada Osservatorio 20, I-10025 Pino Torinese, Italy

${ }^{26}$ Joint Astronomy Center, 660 N. Aohoku Place, University Park, Hilo, HI 96720

${ }^{27}$ Tuorla Observatory, Tuorla 21500 Piikkiö, Finland

${ }^{28}$ NOAO/KPNO, N. Cherry Avenue, P.O. Box 26732, Tucson, AZ 85926

${ }^{29}$ Metsähovi Radio Research Station, 025 $\$ 0$ Kylmala, Finland

${ }^{30}$ Osservatorio Astronomico, Università di Perugia, I-06100 Perugia, Italy

${ }^{31}$ SISSA/ISAS, Via Beirut 2-4, I-34014 Miramare-Grignano (Trieste), Italy

${ }^{32}$ MS 306-388, Jet Propulsion Laboratory, California Institute of Technology, 4800 Oak Grove Drive, Pasadena, CA 91109

${ }^{33}$ Landessternwarte, Heidelberg-Königsstuhl, D-69117 Heidelberg, Germany

${ }^{34}$ Pomona College, Department of Physics \& Astronomy, 610 College Ave., Claremont, CA 91711-6959

${ }^{35}$ Copernicus Astronomical Center, Polish Academy of Science, Warsaw, Poland

Abstract.

We report CGRO, RXTE, ASCA, ROSAT, IUE, HST and ground-based observations of a large flare in 3C 279 in February 1996. X-rays and $\gamma$-rays peaked simultaneously (within one day). We show simultaneous spectral energy distributions prior to and near the flare peak. The $\gamma$ - ray flare was the brightest ever observed in this source. 
In February 1996, the blazar 3C 279 flared dramatically in X-and $\gamma$-rays towards the end of a three-week pointing by CGRO. The intensity of the flare was a factor of $\sim 3$ and $\sim 10$ in $\mathrm{X}$ - and $\gamma$ - rays, respectively. It is the strongest $\gamma$-ray flare ever observed for this object. During one short period of 8 hours, the $\gamma$-ray flux increased by a factor of 2.6 .

Our collaboration carried out observations with CGRO, RXTE, ASCA, ROSAT, IUE, HST, ISO and many ground-based observatories. The most striking result is that the RXTE light curve showed an outburst which was simultaneous with the CGRO-EGRET flare within the temporal resolution of $\sim 1$ day. The optical-UV light curves, which were not as well-sampled during the high energy flare, exhibited smaller variations (factor of $\sim 2$ ) and less obvious correlation. The flux at millimetric wavelengths was quite high, near an historical maximum. ISO observations were obtained in non-standard modes with the photometer and camera; they will be presented elsewhere (Barr et al, in preparation). Lightcurves are shown in Figure 1. A full description is presented in Wehrle et al. [1].

We show (Figure 2) simultaneous spectral energy distributions of 3C 279 prior to and near to the flare peak. In the simplest class of synchrotron selfCompton model, the peak of the gamma ray energy distribution should vary as the square of the height of the optical-IR peak. Thus, in a log-log plot of the broad-band energy distribution (Fig. 2), the displacement of the $\gamma$-ray peak would be twice that of the broad optical-IR peak. Figure 2 shows that the $\gamma$-ray variation is in fact larger than this, which poses problems for the simplest emission models.

\section{A Note on Previous and Current Campaigns:}

Hartman et al. [2] describe the assembly of nearly contemporaneous multiwavelength data, centered on CGRO and GINGA observations, during the June $1991 \gamma$-ray flare [3]. Our first campaign, in which the CGRO, ROSAT, IUE and ground-based observations were coordinated in advance, found the source in a low state during Dec. 1992-Jan. 1993 [4]. The data reported here, using scheduled observations with CGRO, ROSAT, and IUE, independent observations made with HST, performance verification phase observations by ISO, Target Of Opportunity observations with ASCA and RXTE plus many ground-based observatories, are from the second of four campaigns. Our third (coordinated) and fourth (ad hoc) campaigns took place in Dec. 1996-Jan. 1997 and June 1997, involving CGRO, RXTE, ISO and several ground-based observatories.

We are grateful to the satellite and telescope schedulers for making the campaigns possible. A. Wehrle, S. Unwin, E. Pian, and W. Xu acknowledge support from the NASA Long Term Space Astrophysics Program. M. Urry, E. Pian, and J. Pesce acknowledge support from NASA grants NAG8-1037, 
NAG5-2538, and NAG5-9138. M.F. Aller and H.D. Aller acknowledge support from NSF grant AST-9421979.

\section{REFERENCES}

1. Wehrle, A. E. et al. (submitted to Ap.J.) (1997).

2. Hartman, R.C., Collmar, W., von Montigny, C., and Dermer, C.D. 1997 (these proceedings)

3. Hartman, R.C. et al. Ap.J. 461, 698 (1996).

4. Maraschi, L. et al. Ap.J. 435, 91 (1994). 


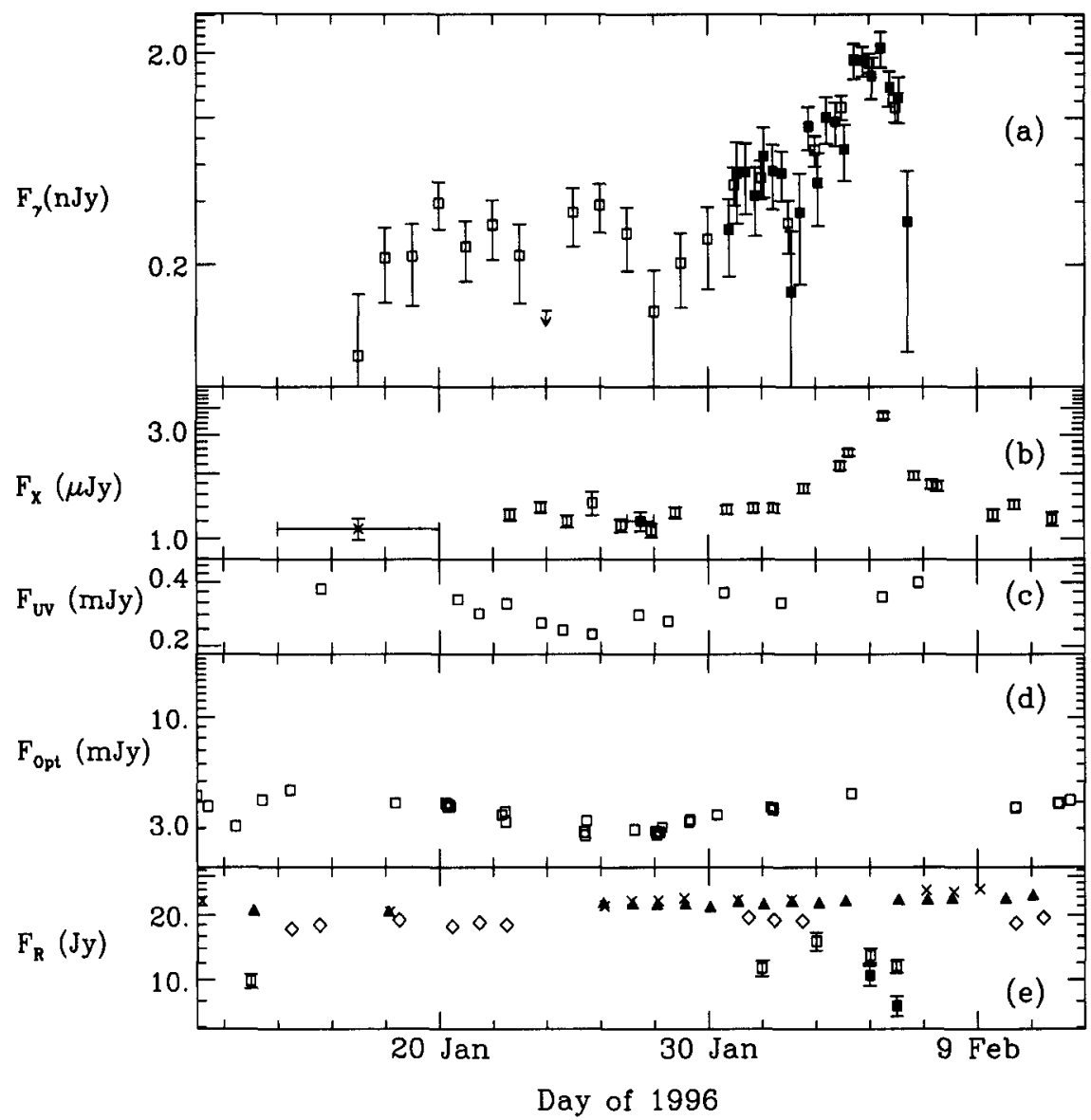

Fig. 1 - Multiwavelength light curves of $3 \mathrm{C} 279$ during the EGRET campaign (1996 January 16 - February 6): (a) EGRET fluxes at $>100 \mathrm{MeV}$ binned within 1 day (open squares) and 8 hours (filled squares); (b) X-ray fluxes at 2 $\mathrm{keV}$ : besides the XTE data (open squares), the isolated ASCA (filled square) and ROSAT-HRI (cross) points are reported with horizontal bars indicating the total duration of the observation; (c) IUE-LWP fluxes at $2600 \AA$, reduced by a factor of 0.44 ; (d) optical data from various ground-based telescopes in the R (open squares). (e) JCMT photometry at $0.45 \mathrm{~mm}$ (filled squares) and $0.8 \mathrm{~mm}$ (open squares), radio data from Metsähovi at $37 \mathrm{GHz}$ (crosses) and $22 \mathrm{GHz}$ (filled triangles), and from UMRAO at $14.5 \mathrm{GHz}$ (open diamonds). Errors, representing 1- $\sigma$ uncertainties, have been reported only when they are bigger than the symbol size. 


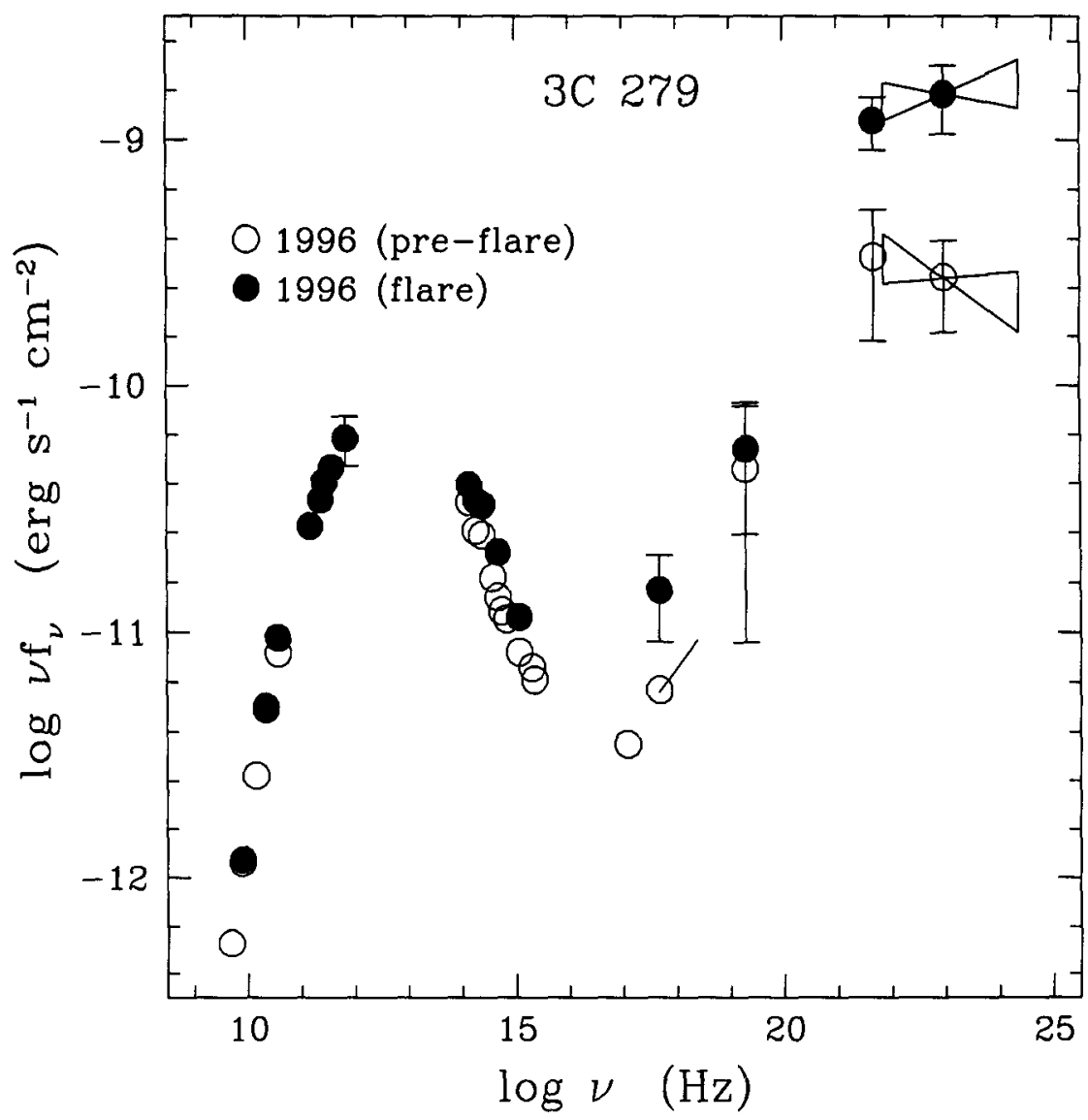

Fig. 2 - Radio-to- $\gamma$-ray energy distribution of $3 \mathrm{C} 279$ in pre-flare (open dots) and flaring state (filled dots) in January-February 1996. The UV, optical and near-IR data have been corrected for Galactic extinction. The slope of the ASCA spectrum $\left(\alpha_{\nu}=0.7\right)$ has been reported normalized at the RXTE point closest in time. The EGRET power-law spectra referring to the 16-30 January and 4-6 February periods are shown, normalized at $0.4 \mathrm{GeV}$. Errors have been reported only when they are bigger than the symbol size. 